\title{
EXPERIENCIAS DE INVESTIGACIÓN-ACCIÓN SOBRE EL APRENDIZAJE POR DESCUBRIMIENTO DE LOS CIRCUITOS ELECTRICOS
}

\author{
MARTIN MUÑoz, A. (1), MUÑoZ ARRABAL, T.(2) y RODRÍGUEZ CARDEÑA, A. (3) \\ (1) Colegio Pabło VI. Ávila. \\ (2) E.U. de Formación del Profesorado. Ávila. \\ (3) Centro de Profesores. Ávila.
}

\section{SUMMARY}

Three experiences on the learning of electric circuits are presented through analysis of those psychopedagogical aspects of learning wich are most relevant in the building of the children's scientific thinking.

\section{INTRODUCCIÓN}

Una de las variables de la función docente en nuestros días es la del profesor-investigador de la clase. El profesor como investigador de su labor cotidiana es una temática que, adecuadamente analizada, puede proporcionar información y datos de gran interés a toda persona ocupada en y preocupada por mejorar los modos de enseñar.

El trabajo que ahora presentamos es el resultado de una experiencia en la que el profesor, consciente de su pa. pel como investigador en la escuela, se ha puesto a ejercer como tal.

Presentamos tres experiencias relativas todas ellas al aprendizaje de los circuitos eléctricos, realizadas en el marco de una Escuela Unitaria Rural (nižos de Ciclo Medio y Ciclo Superior) y de un Centro Completo, en este segundo caso los alumnos pertenecian a Cuarto Curso de EGB.

El total de niños de la Escuela Unitaria que participaron en la experiencia fue de 12 , distribuidos del siguiente modo:

Cuatro niños pertenecientes al Ciclo Medio (dos de $4^{\circ}$ y dos de $5^{\circ}$ curso).

Ocho niños pertenecientes al Ciclo Superior (seis de $7^{\circ}$ $y$ dos de $8^{\circ}$ curso).

El total de niños del centro completo que participaron fue de 28 , todos ellos de $4^{\circ}$ curso.

En cada una de las experiencias, se pretende que los niños construyan, descubriéndolos, los conocimientos necesarios para poder explicar: ¿cómo conectar una bombilla para que luzca?, ¿por dónde van unos circuitos ocultos? y icomo conectar varias bombillas para que luzcan simultáneamente?

Desde una óptica metodológica, no nos interesa tanto el producto o resultado del proceso de aprendizaje co- mo el proceso en sí mismo, es decir, no nos interesa el monto total de lo aprendido, sí el modo de aprender.

En la realización del presente trabajo nos marcamos el siguiente objefivo:

Analizar los aspectos psicopedagógicos del aprendizaje más relevantes en la construcción del pensamiento científico de los niños, bajo la asunción de un modelo pedagógico de "proceso» (Stenhouse 1984), centrándonos en las variaciones que presenta el pensamiento de los niftos según el momento de desarrollo psicológico en que se encuentran.

\section{MÉTODO}

La experiencia tuvo una duración de dos meses y se realiz6 en sesiones de tarde, procurando que cada unidad temática, o una parte significativa de ella, se realizara en una sesión completa de una duración aproximada de dos horas como máximo.

La metodología seguida fue la observación sistemática del proceso de aprendizaje a través de la grabación, en un magnetofón, de los diálogos mantenidos por el profesor con los alumnos y de los alumnos entre si. Los niños realizaron todo el proceso de aprendizaje reunidos en grupos de dos alumnos; cada vez que se finalizaba una parte del aprendizaje, el profesor dialogaba con cada grupo de alumnos a cerca del cómo y el porqué de sus descubrimientos. Así mismo el profesor iba haciendo comentarios sobre como iba discurriendo el proceso.

\section{DESCRIPCIÓN Y ANÁLISIS DE LAS EXPERIENCIAS}

Experiencia $1^{\mathrm{a}}$. ¿Como conectar una bombilla para que luzca?

Se da a los alumnos una pila, una bombilla con su cas 
quillo y dos cables. Se les dice que lo conecten de forma que luzca la bombilla.

Todos los grupos de alumnos lo realizaron correctamente sin emplear mucho tiempo.

Nos parece que los niños descubren y/o tienen ideas elaboradas de los siguientes conceptos:

- La electricidad sale de la pila.

- El camino que sigue la corriente eléctrica tiene que estar cerrado.

El siguiente ejemplo justifica lo dicho anteriormente:

Profesor.- ¿Por qué al poner estos cables junto a una pila la bombilla luce?

Alumno.- Porque la pila tiene electricidad por dentro. Prof.- ¿Cómo le da la pila la electricidad a la bombilia? Alumno.- Pues por el cable.

Prof.- Y si corto el cable, ¿qué pasaría?

Alumno.- Que no funcionaria porque cortas la electricidad.

Prof.- ¿Se puede cortar la electricidad?

Alumno.- Sí. Sólo va hasta donde lo partes.

Sin preguntar a los niños el sentido que llevaba la corriente eléctrica, ellos mismos, apuntaron dos posibilidades (fig. 1 y 2 ).

Dos interpretaciones de los alumnos sobre el sentido de la corriente.

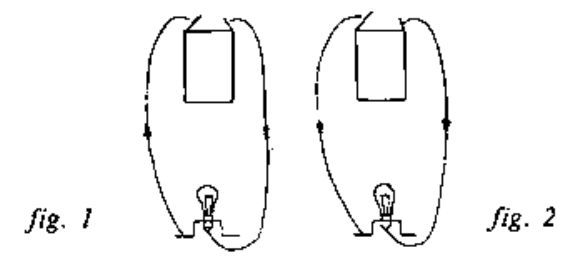

Para profundizar en el pensamiento de los niños sobre cómo circula la corriente eléctrica en un circuito se interrogó, de forma individual, a 20 de ellos. Dieciocho dijeron que la corriente iba según la figura 2 , aduciendo las siguientes razones:

Prof.- ¿Podría ser como en la figura 1 ?

Alumno.- No, porque la electricidad no vuelve a la pila Prof.- ¿Qué has visto tú para saber eso?

Alumno.- Entonces la pila no se gastaría nunca, porque no gastaba energía, la gastaba pero la recuperaba.

Prof." ¿Podría ser como en la figura 1?

Alumno.- No, porque la electricidad ya se gasta en la bombilla y luego ya no vuelve.

Los dos niños que dijeron que la corriente iba según la figura 1 no dieron ninguna explicación del porqué de su elección.

La posibilidad presentada en la figura 2 nos parece más acorde con la experiencia diaria que puede tener el niño, ya que puede haber escuchado expresiones tales como: "Apaga esa luz que gasta mucho». Esas expresio- nes pueden inđucir al niño a creer que la corriente eléctrica sale de la pila y se gasta - se consume - en la bombilla.

Algo que nos llamó profundamente la atención fue el hecho de que todos los niños dijeron que sabian que era asi porque lo habian hecho y lo habian descubierto ellos y que antes de hacerlo no sabian nada sobre el tema.

Prof,- ¿Quién te ha dicho a ti que es asi?

Alumno.- Los hemos averiguado nosotros, lo hemos descubierto.

Prof.- ¿Y antes sabias algo tú de esto?

Alumno.- No.

Los niños a través de sus experiencias, de lo que han escuchado, etc., se han formado la idea de que la electricidad es algo que se gasta en los objetos de uso doméstico (bombilla, plancha, cocina, etc.). Pero quizá sea ésta la primera vez que ellos tienen que manipular e investigar qué conexiones son necesarias para que luzca una bombilla, y esta interacción con los objetos desencadena un proceso mental, que ya estaba latente, y que a la vez que explica el nuevo fenómeno -que en realidad ya estaba explicado- le hace ser consciente de su propio pensamiento.

Como consecuencia de la reflexión anterior, parece ser un error metodológico el pedir a los niños que emitan hipótesis previas a la realización de una experiencia totalmente desconocida para ellos. Nos parece que el nino debería familiarizarse manipulativamente con los objetos de la experiencia para que afloren las estructuras mentales que asistemáticamente se han ido formando a lo largo de su vivir. Es a partir de entonces cuando el niño toma conciencia de sus conocimientos y puede empezar a formular hipótesis, que al verificarlas harán que su pensamiento progrese.

\section{Experiencia $2^{\mathrm{E}}$. ¿Por dónde van los circuitos?}

Dados 7 circuitos construidos con papel de plata y ocultos para los alumnos se les pide a los ninos que, con ayuda de una pila, una bombilla y unos cables, descubran por donde van los circuitos, y dibujen cada uno de ellos con el mínimo número de conexiones. (Fig. 3).

\section{figura 3}

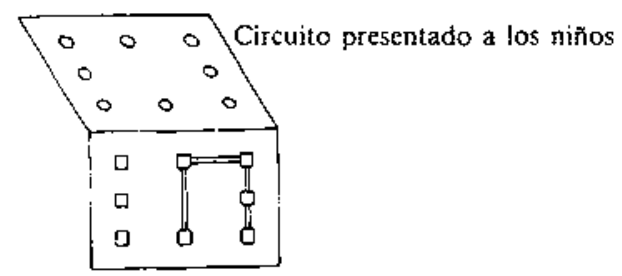

Los niños de Ciclo Medio dominan los procesos manipulativo y gráfico, pero manifiestan dificultades a la hora de explicar verbalmente por donde va el circuito, 
aunque lo hayan representado gráficamente de una manera correcta. (Fig. 4).

Prof.- ¿Cómo habéis sabido que estaban conectados el 4 , el 5 , el 6 y el 10 ?

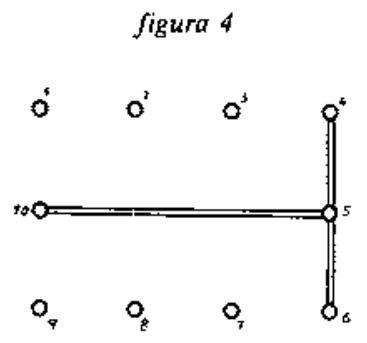

Alumno.- Porque nosotros en cuanto sabiamos que si era el 4 con el 5 , pues ya era el 5 con el 6 y luego ya hemos puesto el 5 con el 10 .

Nos llamó poderosamente la atención el hecho de que más de un $40 \%$ (6 grupos) de los niños del Ciclo, Medio del Centro Completo dominasen la conmutatividad en la verificación de las conexiones (ven la conexíón 1-2, igual a la 2-1 y por tanto ésta última no la verifican). Entre los niños de la Escuela Unitaria ninguno de los pertenecientes al Ciclo Medio manifestó este tipo de pensamiento.

Además estos 6 grupos utilizan una estrategia de seguimiento de un orden en la verifícación de las conexiones. Conectan el orificio 1 con todos los demás, el 2 con todos los demás excepto con el 1 , el 3 con todos los demás excepto con el 1 y el 2 , y asi sucesivamente. Los otros 8 grupos al comenzar a verificar circuitos no dominan la conmutatividad, ni siguen un orden. Cinco grupos de los 8 restantes, a medida que iban verificando circuitos, llegaron a realizarlo con orden en los últimos. Los 3 grupos restantes no manifestaron progreso ni en la conmutatividad ni en el orden.

El progreso realizado por los 5 grupos puede ser debido a dos factores conjuntos e interrelacionados:

a) el contacto manipulativo con la realidad física que les hace ver que si la bombilla luce en la conexión 3-5 también luce en la 5-3, lo cual les conduce a una abstracción reflexionante (Piaget 1977) que les permite ir trascendiendo la realidad concreta, para llegar a conclusiones de carácter general y abstracto.

b) las interacciones con los nintos que desde un principio dominan la conmutatividad, ya que todos los niños pueden moverse libremente por la clase y charlar y observar a sus compañeros.

Pero, ¿por qué los niños de Ciclo Medio de la Escuela Unitaria no han aprendido de sus companeros de Ciclo Superior, los cuales sí dominan la conmutatividad y siguen un orden en las conexiones?
Posiblemente los nif̆os de Ciclo Superior no son «sus iguales», existe un trecho cognitivo dificil de superar. El niño de Ciclo Medio no presenta el nivel de competencia minimo para sacar provecho de la interacción social con sus compañeros de Ciclo Superior (PerretClermont 1984).

Muy probablemente también exista un techo afectivo no siempre fácil de superar por los nitros. Es frecuente que los niños del C. Superior vean a los del C. Medio como los "pequeños" a los que no merece la pena explicar casi nada porque no lo entienden, porque «piensan muy raro». Los nifros del C. Medio ven a los del C. Superior como los mayores, que hacen cosas muy difíciles, pues para eso son los mayores. Sin embargo todos los niftos del C. Medio son iguales, con todos se puede hablar y discutir acerca de cualquier tema.

Los alumnos del C. Superior manifiestan en su totalidad un dominio de la conmutatividad, y siguen un orden en la verificación de las conexiones; además estos alumnos dominan y manejan las operaciones que implican transitividad.

Prof.- ¿Luce el 2 con el 8 ?

Alumno. Si, porque luce el 2 con el 7 , y el 7 con el 8 .

Este manejo de la transitividad se realiza ante la representación gráfica del circuito. Los niños más pequenos necesitan manipular y verificar las conexiones 2-7 y 7-8 para poder llegar a la anterior conclusión. Un paso posterior en la construcción del pensamiento vendría dado por la posibilidad de que las inferencias se produjesen sin apoyo empírica alguna, es decir desde la base del pensamiento abstracto.

\section{Experiencia $3^{\mathrm{B}}$ ¿ ¿Cómo conectar varias bombillas pa- ra que luzcan simultáneamente?}

Se les dió a los alumnos dos bombillas con casquillos, cables y pila y se les pidio que los conectaran de forma que lucieran las dos bombillas a la vez. No se les dijo nada acerca de como debian hacerio.

De los niños de $4^{\circ}$ curso del Centro Completo, cuatro grupos, de catorce, descubren en primer lugar el circuito en paralelo. Al decirles que hay otra forma de realizar un circuito descubren el circuito en serie. EI resto de los grupos, es decir diez, descubren en primer lugar el circuito en serie; al decirles que hay otra forma, construyen el circuito en paralelo con ayuda o aclaraciones de los nintos que lo han realizado correctamente con anterioridad.

De los nifros de la Escuela Unitaria todos ellos descubren el circuito en serie; al decirles que hay otra manera intentan descubrirla pero no lo consiguen. Seguidamente el profesor dibuja en el encerado el diagrama del circuito en paralelo para que los propios alumnos realicen el montaje.

Una vez que todos los niffos han realizado los montajes en serie y en paralelo se les dan tres bombillas para- 
que las conecten de modo que luzcan a la vez. Todos los niffos realizaron los montajes sin problemas, haciéndolos en serie y en paralelo y ninguno mixto.

Con los circuitos de las tres bombillas funcionando, se hicieron las siguientes preguntas:

¿Qué ocurre al aflojar una bombilla?

¿Por qué?

Todos los niños de C. Medio dan explicaciones adecuadas de porqué en un circuito en serie, al aflojar una bombilla, las demás se apagan.

Alumno.- Porque no puede pasar la electricidad porque sólo va unida por un cable.

Esta explicación no está reñida con su concepción errónea del sentido que sigue la corriente eléctrica en un circuito simple. La generalización del camino que sigue la corriente eléctrica en un circuito simple a otros más complejos se hace sin ninguna dificultad. Según el pensamiento infantil la corriente siempre se gasta en las bombillas, lo mismo da que haya una que varias. Habrá que esperar, en antos posteriores, a que su intento de explicación de ciertos fenómenos magnéticos, al cambiar las conexiones, sean las que obliguen a reconsiderar su modelo de movimiento de la corriente eléctrica.

Los alumnos de C. Medio dan explicaciones correctas sobre el porqué, en un circuito en paralelo, al aflojar cualquier bombilla las restantes brillan más.

Alumno.- La electricidad que estaba en ésa, pasa a las otras dos que quedan y entonces lucen más al tener más energia.

Prof.- ¿Y si aflojamos dos?

Alumno.- Pues que la energía que estaba en las dos pasa a la última que quedaba y luce más.

Al preguntar a los alumnos de C. Medio sobre si la pila da siempre la misma cantidad de electricidad, tanto si está conectada a una sola bombilla como a varias, se obtienen respuestas dispares. Para unos si que da la misma cantidad de electricidad, pero para otros no.

Todos estos ninos muestran un pensamiento de consistencia muy frágil, se dejan arrastrar muy fácilmente por el pensamiento de sus compañeros tanto en un sentido como en otro. Posiblemente su pensamiento ya empieza a dominar el tipo de operaciones intelectuales que supone la compensación: número de bombillas $x$ brillo de cada una de ellas; compensación casi análoga a: anchura de un recipiente $x$ altura de un liquido en dicho recipiente.

Creemos que los niños deben reconstruir sus adquisiciones del pensamiento para cada nuevo aspecto de la realidad; la electricidad es un aspecto de la realidad bastante novedoso para ellos y creemos que deben reconstruir propiedades que ya estaban dominadas en otros campos de la realidad física. La transferencia del aprendizaje no se suele dar de un modo inmediato; los ni- fos suelen tardar bastante tiempo en transferir los aprendizajes de un terreno a otro y esto produce en los profesores una sensación de fracaso poco grata. Los ninos, y posiblemente los adultos, necesitan un tiem. po para readaptar su estructuras de pernsamiento a la nueva situación.

\section{CONCLUSIONES}

\section{Aspectos psicopedagógicos}

De acuerdo con nuestro objetivo de analizar los aspectos psicopedagógicos más relevantes en el proceso de construcción del pensamiento científico de los niños, en el terreno de los circuitos eléctricos, centrándonos en las variaciones que presenta su pensamiento según el momento de desarrollo psicológico en el que se encuentran, elaboramos las siguientes conclusiones.

Las características del pensamiento de los nir̃os de $\mathrm{Ci}$ clo Medio y Ciclo Superior se ajustan, en el terreno de los circuitos eléctricos, a las características generales del pensamiento infantil --para estas edades - descritas por Piaget y otros autores en diversas obras.

\section{Hay uno graduación desde el Ciclo Medio hasta el último curso del Ciclo Superior en separar las percep- ciones directas de lo que se puede inferir de esa realidad.}

Hay un continuo con dos extremos nítidos; en uno de ellos estarían los niños cuyas conclusiones estarían basadas únicamente en la percepción; en el otro extremo se situarían aquellos alumnos cuyas conclusiones no se basan en lo que parece ser, sino que trascienden la realidad. Son niños que diferencian entre lo fenoménico $o$ aparente y lo real o verdadero.

a) Los alumnos de todos los ciclos, tanto a través de las experiencias realizadas, como de sus aprendizajes espontáneos y de las interacciones con otras personas -adultos y niños-, son capaces de trascender la realidad y llegar a las siguientes conclusiones sobre un circuito eléctrico simple:

- La electricidad sale de la pila.

- La electricidad va por los cables.

- El camino debe estar cerrado.

Decimos que el niño trasciende la realidad porque él no ve la electricidad salir de la pila, ni circular por los cables. El niño debe imaginarse la electricidad, él no la ve, sólo ve sus efectos y él se construye un modelo que como hemos visto anteriormente es alternativo, que se ajusta a «su realidad», a sus ideas originadas de un modo espontáneo.

b) En la experiencia que consistía en inferir qué circuitos eléctricos había en las cartulinas se ha mostrado una amplia gama de modos de realización.

Todos los ninos, tanto los de Ciclo Medio como los de Ciclo Superior, realizaron satisfactoriamente la ex- 
periencia mencionada. Ha sido en el modo de realización y en las habilidades cognitivas puestas en marcha en Io que ha habido diferencias más significativas. Las habilidades más eficaces han sido:

- Ver la conexión 1-2 igual que la 2-1. De este modo hay una considerable economía de tiempo, pero no es sólo la economía de tiempo lo importante, sino también el modo en que opera el conocimiento.

- Verificar las conexiones siguiendo un orden en las mismas. Si se prueba el 1 con todos los demás, el 2 con todos los demás menos con el 1, etc. no se corre el riesgo de olvidar ningún orificio para verificar las conexiones.

Los alumnos más pequeños, no tanto en edad sino sobre todo en desarrollo cognitivo, necesitan la realidad; a medida que crece su desarrollo cognitivo ya no necesitan toda la realidad, pueden trascenderla desde su visualización gráfica, y los más adelantados ya no necesitan ni siquiera ésta, pueden hacerlo desde la verbalización de esa realidad. El niño más pequeño para comprobar las conexiones 3-5-7 debe poner la bombilla entre el 3 y el 5 , entre el 5 y el 7 y entre el 3 y el 7 . EI niño más adelantado no necesita comprobar la conexión 3-7 si ya ha comprobado la 3-5 y la 5-7; pero no sólo esto, sino que puede hacer lo mismo de un modo visual -y no manipulativo- ante el circuito. En un último estadio el niño lo puede hacer escuchando las caracteristicas de las conexiones, sin estar ni frente al circuito real ni a su representación gráfica. Va habiendo por tanto, un progresivo distanciamiento de la realidad percibida.

\section{II. - Hemos observado en los niños una progresión en lo que se ha llamado "Centración frente a descentración.}

Como apunta Flavell, el niño más pequeño confía excesivamente en sus entradas perceptivas, pero a medida que va siendo mayor el nif̃o stambién atiende cuidadosamente a la entrada perceptiva durante toda la realización de la tarea, aunque en última instancia se dé cuenta de que la tarea requiere en realidad un juicio conceptual en vez de perceptivo. Además, es capaz de distribuir su atención de un modo más flexible, equilibrado y, en términos generales, más adaptado a la tarea. El niño pequeño es más proclive a concentrar o centrar (de ahí el término centración) sú atención ex- clusivamente en un solo rasgo o en una sola parte del estímulo que resulte especialmente destacado e interesante para él, descuidando, por tanto, otros rasgos de la tarea igualmente relevantes.

En cambio el nir̃o mayor suele efectuar un análisis perceptivo de toda la situación, más equilibrado, descentrado (de ahí descentración). De esta forma logra una visión más amplia e inclusiva de la totalidad del estímulo. En definitiva, es más probable que perciba y tenga en cuenta todos los datos perceptivos relevantes. (Flavell 1984, El desarrollo cognitivo, pag. 97-98).

Todos estos aspectos del desarrollo cognitivo infantil se ponen de manifiesto en las conversaciones mantenidas con los niños durante y después de la realización de algunas experiencias.

La experiencia realizada tanto con los circuitos en serie como en paralelo no estaba únicamente encaminada al montaje de esos dos circuitos, sino que también iba encaminada a que el nifno adquiriese una comprensión de las características diferenciadoras de los dos tipos de circuitos.

Al interrogar a los alumnos más pequeftos sobre si, en un circuito en paralelo, la cantidad de electricidad suministrada por la pila es independiente del número de bombillas conectadas, y sobre si la cantidad de electricidad permanece constante para un periodo de tiempo relativamente breve ( 5 minutos) se obtiene una respuesta negativa. Sus percepciones están únicamente centradas en el brillo de las bombillas. A medida que los ninos progresan en su desarrollo cognitivo, sus percepciones van paulatinamente descentrándose de ese único punto de vista y van incluyendo un factor más: el número de bombillas. Con la inclusión de este nuevo factor el nifno empieza a realizar descentraciones y por lo tanto compensaciones; compensa el mayor número de bombillas con el menor brillo de cada una de ellas. Es como si dijese: número de bombillas $x$ brillo de cada una de ellas $=$ constante.

En este aspecto, como en los anteriores, hay una completa graduación del modo de pensar de los niños. La amplitud de la distribución va desde aquellos alumnos que se centran en un solo aspecto, hasta aquéllos que tienen presentes todos los aspectos sopesando cada uno de ellos; asi mismo hay multitud de casos intermedios que cubren casi todos los aspectos posibles.

\section{REFERENCIAS BIBLIOGRAFICAS}

FLAVELL, 1984. El desarrollo cognitivo. (Visor: Madrid).

PERRET-CLERMONT, A.N., 1984. La construcción de la inteligencia en la interacción social. (Visor: Madrid).

PIAGET, J., 1977. Investigaciones sobre la abstracción reflexionante. (Huemul S.A.: Buenos Aires).

STENHOUSE, L., 1984. Investigación y desarrollo del curriculo. (Morata: Madrid). 